\title{
Assessment of Visual Motor Integration in Children with Developmental Coordination Disorder in Indian Context
}

\author{
Ganapathy Sankar $\mathbf{U}^{1}$ and Monisha $\mathbf{R}^{2 *}$ \\ ${ }^{1}$ SRM College of Occupational Therapy, SRM Institute of Science and Technology, India \\ ${ }^{2} S R M$ College of Physiotherapy, India \\ *Corresponding author: Monisha R, Assistant Professor, SRM College of Physiotherapy, Chennai, India, Email: monishaphysio186@gmail.com
}

Submission: 海August 16, 2018; Published: 眥 September 07, 2018

\begin{abstract}
Introduction: Visual Proprioception utilizes a visual form of information to communication and to share knowledge, helps in thinking and creative processes. Visual-motor coordination is needed to perform all the activity of daily living and it is a foundational skill required for all the children for academic achievement.

Methodology: The study has been initiated in June 14-2018, after getting informed consent signed, Ten children referred for their motor problems in home and in school to a rehabilitation clinic in and around Chennai has been selected in this study, Movement assessment battery for children (ABC) was administered to the study sample and 5 children have impairment score below 5 th percentile.

Result: Children with DCD show significant poor performance in the entire test of Berry VMI test and these Children with DCD appears to have problem in proprioception and motor programming.

Discussion: Overall, the problems of children with DCD become most pronounced when their academic performance has been affected because of the poor visual motor integration. They have poor eye- hand coordination when writing examination and they were reported to have difficulty in fixing the paper to the desk in order to create the stable environment for writing the test. Children's with DCD fails to do so.
\end{abstract}

Conclusion: Children with DCD appears to have problem in Visual motor integration and because of this these children have poor performance in all perceptual task, hence the study concludes that difficulty in visual-motor integration contribute to poor academic performance.

Keywords: Visual motor integration; DCD, Berry scale; MABC

\section{Introduction}

The motor activity, children execute in everyday life arises from the integration of work and processing acuity of many systems [1-4]. The motor system controls the processing and execution of motor task and sequencing of movements according to sensory input. For the motor system to produce integrated movements, cognitive system and the integration of attention, motivation and planning are in need. Visual proprioception is needed to provide feedback regarding the position of body and its segments and it helps in analyzing the distinction between performance and sequencing of motor events, the proprioceptors which carry afferent information, proprioceptive judgment of limb position can be achieved purely through sensory matching and body-centered spatial judgments posits difficulty for children with DCD [5].

Literature search has identified that children's difficulty in motor coordination and sequencing of events is because of damage to specific group of neuron or brain regions. DCD can result if a child experiences difficulty in proprioception, programming of motor events and timing and in sequencing motor events [6]. The problems experienced by children with DCD in activity of daily living and in academic performance, however their IQ is reported to be higher than $70 \%$ as compared to their age matched peer groups, these, difficulty are believed to be originating from defects in neuro transmitter or in the receptor system.

The children with DCD in previous research trial revealed significantly poorer performance than control children in visuoproprioceptive matching tasks. They had poorer coordination on cross modal judgements; here visual guidance is in need to accomplish the proprioceptive perception of limb position, children in the experimental group seemed to have particular difficulty in cross model judgment [7]. Visual Proprioception 
utilizes a visual form of information to communication and to share knowledge, helps in thinking and creative processes. Visual-motor coordination is needed to perform all the activity of daily living and it is a foundational skill required for all the children for academic achievement.

Coordinated fine motor movements and visual perception skill is highly demanding to perform activity of daily living. In children with DCD acquisition of grapho-motor skills are highly challenging because of the deficit in visual proprioception and motor task execution. The visual-motor coordination is the matching of motor output with visual input and the coordination between this two [8] It is a process of transmitting integrated information possessed by the visual system and integrated into action by processing the information into motor action.

Early coupling of visual-motor task has to be developed in children to execute the activity of daily living as like the peer groups. Visual-motor integration is an important factor contributing to academic achievement. Evidences on correlation between VMI and academic achievement in DCD children are limited with higher perception of controversial results. None of the study has been done in Indian population. Poor performance in primary schooling has been reported in children with poor visual-motor integration. If left untreated these children will carry on these difficulties into adolescence and have poor self-esteem and impaired relationship with peer groups.

Physical and occupational therapist working in children's with DCD identified various factors affecting children academic performance and activity of daily living. Factors includes psychological aspects, cognitive aspects, visual perception, VMI Objective of the therapy sessions is to facilitate participation in daily activities such as self-care and play [9]. Thus, the aim of the study is to investigate the visual-motor coordination in children with DCD and to analyze the impact of VMI in academic achievement of children as compared to their peer group.

\section{Method}

The study has been initiated in June 14- 2018, after getting informed consent signed, ten children referred for their motor problems in home and in school to a rehabilitation clinic in and around Chennai has been selected in this study. Parents were explained about assessment procedure and Children with the age group of 7 years and not a diagnosed case of any Cardio vascular disorder, diabetes and hypertension. Pervasive developmental disorder and didn't have any indicated neurological deficit or physical impairment. IQ score of above 70 on intelligence test. Out of ten, 6 children scored greater than 70 on intelligence test were included in this study. Movement assessment battery for children (ABC) was administered to the study sample and 5 children have impairment score below $5^{\text {th }}$ percentile and they are the definite case of DCD with predominant motor problem and the remaining 1 child have completed MABC didn't shows impairment under motor domain, hence excluded out of the study. Therefore a total of 5 had participated in this study.
To find the difference in these 5 children for their Visual-motor integration, Beery-Buktenia developmental test of visual motor integration has been selected. It is the gold standard assessment tool used in preschool children to evaluate the VMI and study aims to obtain needed services and therapeutic intervention for individual those exhibit difficulties [10,11]. Early assessment of these children will help them not to carry on their difficulties and to avoid psychosocial symptoms in the future.

Beery VMI contains 30 items it takes 15 minutes to complete the administration process, here the children have been asked to copy the geometric shapes with paper and pencil. Children's answers to the test can be scored correct or wrong, based on the manual. Score of 1 is given for correct answer and score 0 is given for incorrect answer. Minimum score obtained in the test result is 0 and the maximum score is 30 . The advantage of using this test in Indian population is the assessment tool lacks cultural bias.

Pure motor and pure visual problems can be isolated in all the children with the help of Beery VMI Visual perception test and beery VMI motor coordination test. Instructions have been given in Tamil to all the children included in the study. The second objective of the study is to evaluate the correlation between children's VMI and academic performance. School mark sheet for each child has been evaluated to know their academic performance and the teachers has been provided with academic performance index, it rates the performance of each child in 0-100-point rating scale. Marks from rank card can be converted to 100 and it can be used.

\section{Data Analysis}

Descriptive statistics of primary outcome measures VMI and Academic performance index (Table 1).

Table 1: Number of children with DCD and their Mean and standard deviation of VMI and academic performance index.

\begin{tabular}{|c|c|c|c|c|}
\hline S.NO. & Variables & N & Mean & SD \\
\hline 1. & VMI & 5 & 70.01 & 13 \\
\hline 2. & Academic performance index & 5 & 60 & 10 \\
\hline 3. & Academic performance in rank card & 5 & 50.8 & 8.6 \\
\hline
\end{tabular}

Pearson product movement correlation has been used to compare the academic performance index scoring provided by teachers and performance calculated by converting the marks obtained in school rank card to 100. Strong correlation exists between this two and school rank card is the objective and reliable way of assessing the academic performance as teachers report needs further validation because of the existence of various confounding extraneous factors (Figure 1\&2, Tables 2-5).

Table 2: Descriptive statistics.

\begin{tabular}{|c|c|c|c|}
\hline & Mean & Std. Deviation & N \\
\hline Academic perform index & 11 & 2.345 & 5 \\
\hline School rank card & 12 & 2.739 & 5 \\
\hline
\end{tabular}




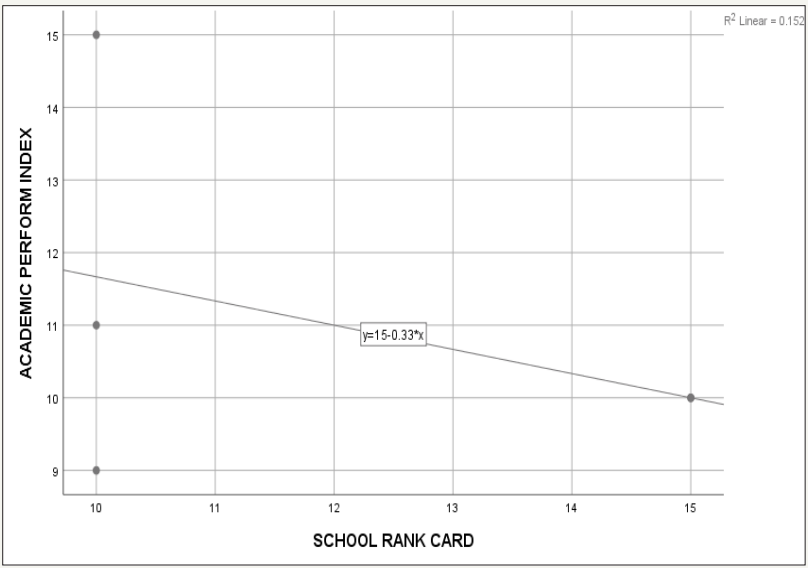

Figure 1: Correlation between academic performance index by school rank card and teachers scoring scale.

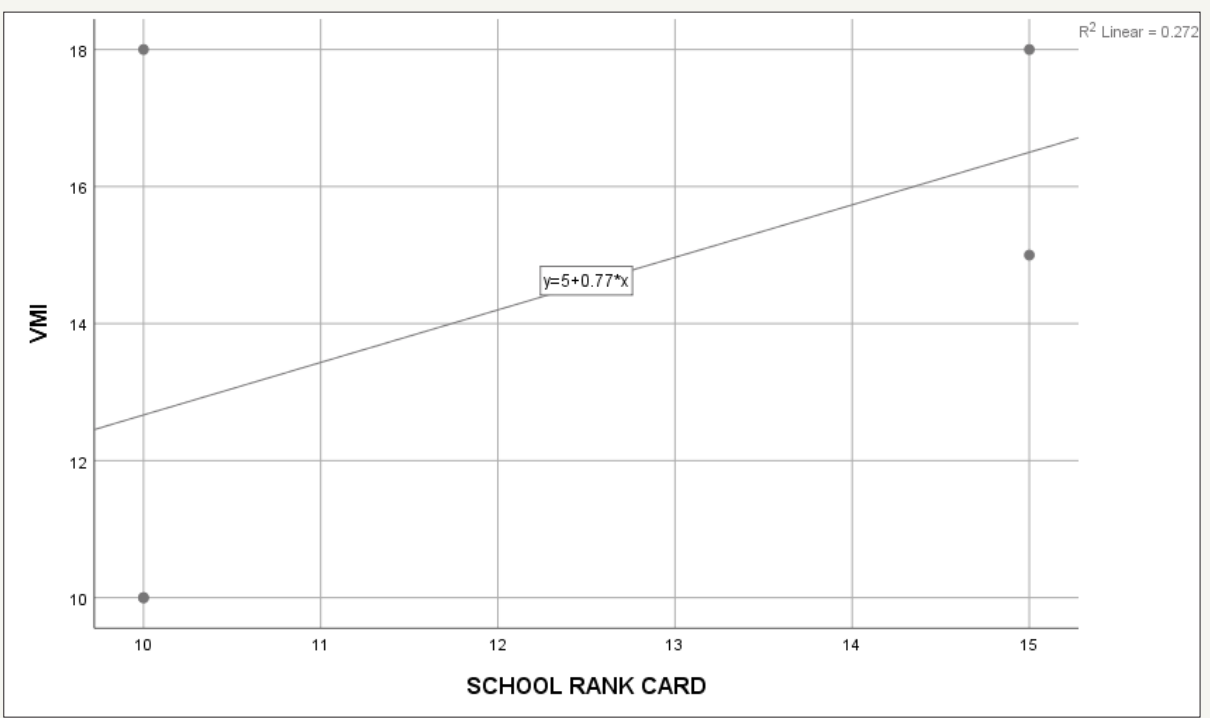

Figure 2: Correlation between VMI and academic performance from report card.

Table 3: Correlations between academic performance index by teachers and the rank card.

\begin{tabular}{|c|c|c|c|}
\hline \multirow{2}{*}{ Academic perform index } & & Academic Perform Index & School Rank Card \\
\hline & Pearson Correlation & -0.389 \\
\cline { 2 - 4 } & Sig. (2-tailed) & 5 & 0.517 \\
\cline { 2 - 4 } & $\mathrm{N}$ & -0.389 & 5 \\
\hline \multirow{2}{*}{ School rank card } & Pearson Correlation & 0.517 & 1 \\
\cline { 2 - 4 } & Sig. (2-tailed) & 5 & 5 \\
\hline
\end{tabular}

Table 4: Descriptive statistics.

\begin{tabular}{|c|c|c|c|}
\hline & Mean & Std. Deviation & N \\
\hline School rank card & 12 & 2.739 & 5 \\
\hline VMI & 14.2 & 4.025 & 5 \\
\hline
\end{tabular}

Table 5: Correlations.

\begin{tabular}{|l|c|c|c|}
\hline & & School Rank Card & VMI \\
\hline \multirow{3}{*}{ School rank card } & Pearson Correlation & 1 & 0.522 \\
\cline { 2 - 4 } & Sig. (2-tailed) & 0.367 \\
\cline { 2 - 4 } & $\mathrm{N}$ & 5 & 5 \\
\hline
\end{tabular}




\begin{tabular}{|c|c|c|c|}
\hline \multirow{3}{*}{ VMI } & Pearson Correlation & 0.522 \\
\cline { 2 - 4 } & Sig. (2-tailed) & 0.367 & 5 \\
\cline { 2 - 4 } & $\mathrm{N}$ & 5 & 5 \\
\hline
\end{tabular}

\section{Result}

Children with DCD show significant poor performance in the entire test of Berry VMI test and these Children with DCD appears to have problem in proprioception and motor programming, significant correlation has been obtained in children with DCD when their academic performance and VMI has been compared.

\section{Discussion}

This study aims to analyze whether children with DCD have problem with Visual motor integration and whether this will affect their academic performance. Wilson and McKenzie (2001), in previous studies analyzed that general isolated aspects of visual, proprioceptive or tactile skills were impaired in children with DCD. They experience difficulty in perceiving the shape of figures and numbers that were hidden in a complicated background and they show poor performance in arranging alphabets in order from $A$ to $\mathrm{Z}$, there is a mismatch between B and D, U and C. They also had difficulty in discriminating different size. visual spatial impairments for children with DCD has been identified by previous researchersHolman, deficit has been reported when they were asked to join the presented straight lines or dotes, the sample selected in trial were from age group of 6 and it has been highlighted that the inclusion criteria is not responsible for this conflicting result. Children with DCD find difficulty in visual perception.

Overall, the problems of children with DCD become most pronounced when their academic performance has been affected because of the poor visual motor integration. They have poor eye- hand coordination when writing examination and they were reported to have difficulty in fixing the paper to the desk in order to create the stable environment for writing the test. Children's with DCD fails to do so. This high percentage of difficulty in achieving visual motor integration is needed to be assessed in early years to optimize therapy and to avoid the negative consequences into adulthood. But no study had done analyzing the relationship between DCD identified by MABC and proprioceptive skill, many research trials failed to identify the cause and the relationship between perceptual skill and motor coordination disorder. Therefore, the findings of this study have identified that children with DCD have problems in visual, proprioceptive and Visual motor integration to achieve academic excellence.

\section{Conclusion}

Children with DCD appears to have problem in Visual motor integration and because of this these children have poor performance in all perceptual task, hence the study concludes that difficulty in visual-motor integration contribute to poor academic performance.

\section{Limitation and Recommendation}

There has been no single gold standard assessment tool in identifying children with DCD. There is inconsistency in motor test used in identifying children with DCD and all the assessment tool ends in hypothesis. No one test, however can accurately identify DCD children or replace the clinical reasoning of a therapist who examines multiple sources of information about a child.

\section{References}

1. American Psychiatric Association (1987) Diagnostic and statistical manual of mental disorders. ( $\left.3^{\text {rd }} \mathrm{edn}\right)$, Washington, USA.

2. American Psychiatric Association, Bairstow PJ, Laszlo JI (1981) Kinaesthetic sensitivity to passive movements and its relationship to motor development and motor control. Developmental Medicine and Child Neurology 23(5): 606-616.

3. Ganapathy SU (2018) The developmental coordination disorder at kattupakkam. IOSR Journal of Pharmacy 8(2): 49-52.

4. Doyle AJR, Elliott JM, Connolly KJ (1986) Measurement of kinaesthetic sensitivity. Developmental Medicine and Child Neurology 28(2): 188193.

5. Ganapathy SU, Saritha S (2011) The prevalence of developmental coordination disorder at kattankulthur. Indian Journal of Physiotherapy \& Occupational Therapy 5(1): 63-65.

6. Elliott JM, Connolly KJ, Doyle AJR (1988) Development of kinaesthetic sensitivity and motor performance in children. Developmental Medicine and Child Neurology 30(1): 80-92.

7. Hulme C, Biggerstaff A, Moran G, McKinley I (1982a) Visual, kinaesthetic and cross-modal judgements of length by normal and clumsy children. Developmental Medicine and Child Neurology 24(4): 461-471.

8. Smart A, Moran G (1982b) Visual perceptual deficits in clumsy children Neuropsychologia 20(4): 475-481.

9. Huynh H, Feldt LS (1970) Conditions under which mean square ratios in repeated measure designs have exact F-distributions. Journal of the American Statistical Association 65(332): 1582-1589.

10. Jongmans M, Henderson S, de Vries L, Dubowitz L (1993) Duration of periventricular densities in preterm infants and neurological outcome at 6 years of age. Archives of Disease in Childhood 69: 9-13.

11. Polatajko HJ, Macnab JJ, Anstett B, Malloy-Miller T, Murphy K, et al. (1995) A clinical trial of the process-oriented treatment approach for children with developmental coordination disorder. Dev Med Child Neurol 37(4): 310-319. 
(c) (i) Creative Commons Attribution 4.0

For possible submissions Click Here

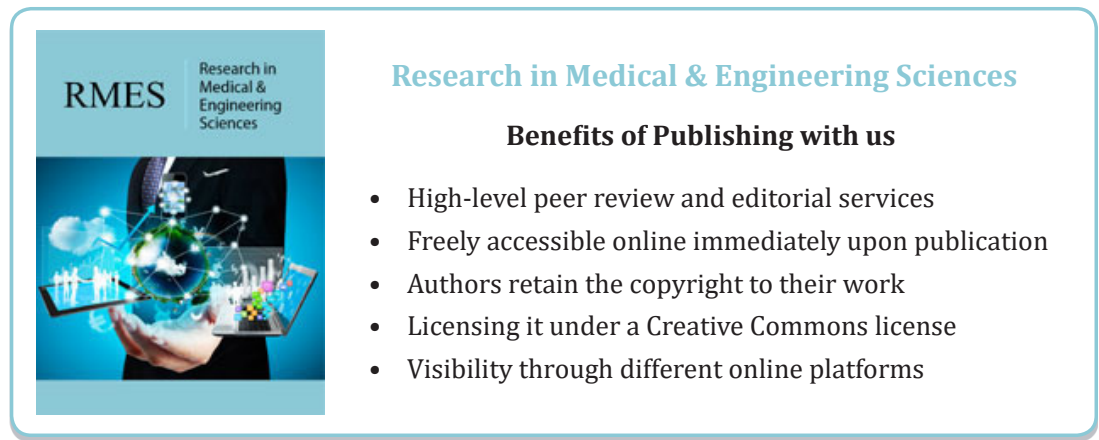

